\title{
Parvalbumin-Containing Chandelier and Basket Cell Boutons Have Distinctive Modes of Maturation in Monkey Prefrontal Cortex
}

\author{
Kenneth N. Fish, ${ }^{1 \star}$ Gil D. Hoftman, ${ }^{2,3 *}$ Wasiq Sheikh, ${ }^{1}$ Michael Kitchens, ${ }^{1}$ and David A. Lewis ${ }^{1,3}$ \\ ${ }^{1}$ Department of Psychiatry, ${ }^{2}$ Medical Scientist Training Program, and ${ }^{3}$ Department of Neuroscience, University of Pittsburgh School of Medicine, \\ Pittsburgh, Pennsylvania 15213
}

\begin{abstract}
Parvalbumin (PV)-containing cortical GABA neurons include chandelier cells (PVChCs) and basket cells (PVBCs), which innervate the axon initial segment (AIS) and soma/proximal dendrites of pyramidal cells, respectively. In monkey prefrontal cortex (PFC), the density of PVChC axon cartridges detectable by PV immunoreactivity peaks prior to the onset of puberty before declining markedly to adult levels, whereas the density of PV-immunoreactive (IR) puncta (presumed PVBC boutons) increases during adolescence. These inverse developmental changes in bouton density could explain why an electron microscopy study found no change in the density of symmetric, presumably GABAergic, synapses between infancy and adulthood in monkey PFC. Alternatively, the inverse developmental trajectories of PVChC and PVBC boutons could represent cell type-specific differences in the maturation of PV protein levels. To differentiate between these two alternatives, multilabel confocal microscopy was used to quantify the number of PVChC and PVBC boutons per pyramidal neuron in the PFC of 3-month-old and adult monkeys. The mean number of PVChC boutons per pyramidal neuron AIS was, significantly, $32 \%$ lower in adult compared with 3-month-old monkeys, whereas the density of PVBC boutons per pyramidal neuron did not differ between age groups. In contrast, relative levels of PV protein were approximately twofold higher in PVBC boutons in adult animals, whereas PV levels in PVChC boutons did not differ between age groups. These findings suggest cell type-specific mechanisms of maturation of PV-containing GABAergic boutons in monkey PFC.
\end{abstract}

\section{Introduction}

The postnatal development of GABAergic circuitry in the monkey prefrontal cortex (PFC) is a protracted process that extends into early adulthood (Hoftman and Lewis, 2011). This process includes the establishment and maturation of precise connections among neuronal populations. For example, the axons of chandelier (axoaxonic) cells (ChCs) exclusively target the axon initial segment (AIS) of pyramidal neurons (Szentagothai and Arbib, 1974); the convergence of axons from multiple ChCs onto a single pyramidal neuron AIS forms a distinctive, vertically oriented array of boutons termed a cartridge (Lewis and Lund, 1990). Many cortical ChCs contain

\footnotetext{
Received Jan. 18, 2013; revised March 12, 2013; accepted April 4, 2013.

Author contributions: K.N.F., G.H., and D.A.L. designed research; K.N.F., G.H., W.S., and M.K. performed research; K.N.F. and G.H. analyzed data; K.N.F., G.H., and D.A.L. wrote the paper.

This work was supported by NIMH Grants MH096985 (K.N.F.), MH093079 (G.H.), and MH051234 (D.A.L.). The content is solely the responsibility of the authors and does not necessarily represent the official views of the NIMH or the NIH. We are extremely grateful to Dr. Robert Sweet for his help in the sampling design and Lindsey McClement for her technical assistance. D.A.L. currently receives investigator-initiated research support from Bristol-Myers Squibb and Pfizer, and in 2010-2012 served as a consultant in the areas of target identification and validation and new compound development to Bristol-Myers Squibb and Concert Pharmaceuticals. The remaining authors declare no competing financial interests.

*K.N.F. and G.H. contributed equally to this work.

Correspondence should be addressed to Dr. David A. Lewis, Department of Psychiatry, Western Psychiatric Institute and Clinic, University of Pittsburgh, Biomedical Science Tower, Room W1651, Pittsburgh, PA 15213. E-mail: lewisda@upmc.edu.

DOI:10.1523/JNEUROSCI.0306-13.2013

Copyright $\odot 2013$ the authors $\quad 0270-6474 / 13 / 338352-07 \$ 15.00 / 0$
}

the calcium-binding protein parvalbumin (PVChC) (DeFelipe et al., 1989), and the density of cartridges detectable by PV immunoreactivity decreases between 3 months and adulthood in monkey PFC (Cruz et al., 2003). In contrast, the density of PVimmunoreactive (IR) boutons that do not belong to cartridges, and which presumably arise from PV-containing basket cells (PVBCs), significantly increases between these same ages (Erickson and Lewis, 2002). Both of these findings appear to conflict with the results of other studies indicating that the overall densities of GABAergic synapses in monkey PFC do not undergo postnatal developmental refinements. Specifically, the density of all symmetric (putatively inhibitory) synapses does not change between 3 months and adulthood (Bourgeois et al., 1994), and the density of GABA membrane transporter 1 (GAT1)-IR puncta appears to remain constant (Erickson and Lewis, 2002).

This apparent conflict might be resolved by opposite but equivalent changes in the densities of PVChC and PVBC boutons across postnatal development, such that the overall density of inhibitory boutons and synapses does not change. Alternatively, the apparent changes with age in the densities of PVChC cartridges and PVBC boutons could reflect inverse developmental shifts in bouton levels of PV protein such that PVChC and PVBC boutons become less and more detectable with age, respectively. To discriminate among these alternatives, we used quantitative, multilabel, fluorescence confocal microscopy to assess the density of, and relative PV protein 
levels in, the boutons of PVChCs and PVBCs in the PFC of 3-month-old and adult monkeys.

\section{Materials and Methods}

Animals. Macaque (Macaca fascicularis) monkeys (3-month-old, 3 females) and adult (156-192-month-old; 2 females, 1 male) were anesthetized and then perfused transcardially as previously described (Cruz et al., 2003). Brains were immediately removed, and coronal blocks (5-6 $\mathrm{mm}$ thick) were cut and stored in a cryoprotectant solution at $-30^{\circ} \mathrm{C}$ (Cruz et al., 2003). Sections $(40 \mu \mathrm{m})$ were exhaustively cut from left hemisphere blocks containing the entire rostral-caudal extent of the principal sulcus (area 46). All experimental procedures were conducted in accordance with the NIH Guide for the Care of and Use of Laboratory Animals and with the approval from the University of Pittsburgh's Institutional Animal Care and Use Committee.

Antibodies and immunocytochemistry. Two sections per monkey, 1 $\mathrm{mm}$ apart, were taken from the middle one-third of the principal sulcus (Fig. 1) and processed as follows. Sections were permeabilized with $0.3 \%$ Triton X-100 in PBS for 30 min at room temperature (RT), rinsed, and blocked with $20 \%$ donkey serum for $2 \mathrm{~h}$ at RT. Sections were then incubated for $\sim 72 \mathrm{~h}$ at $4^{\circ} \mathrm{C}$ in PBS containing 3\% donkey serum and the following antibodies: PV (sheep, R\&D Systems; 1:100) (Kagi et al., 1987) and the vesicular GABA transporter [(vGAT) guinea pig, Synaptic Systems; 1:500] (Guo et al., 2009), which concentrates in GABAergic boutons (Chaudhry et al., 1998), to identify boutons; the $\mathrm{GABA}_{\mathrm{A}}$ receptor subunit $\gamma 2[(\gamma 2)$ rabbit, Synaptic Systems; 1:400] (Fritschy and Mohler, 1995) to identify postsynaptic sites of GABA boutons; and ankyrin-G [(AnkG) mouse, Santa Cruz Biotechnology; 1:100] (Wang and Sun, 2012) and neuronal nuclei [(NeuN) mouse, Millipore; 1:500] (Mullen et al., 1992) to identify the AIS and soma, respectively, of pyramidal neurons. Sections were then rinsed for $2 \mathrm{~h}$ in PBS, incubated with secondary antibodies (donkey; 1:500) conjugated to Alexa 405, 488, 568, and 647 (Invitrogen), or biotin (Fitzgerald) for $24 \mathrm{~h}$ in $\mathrm{PBS}$ at $4^{\circ} \mathrm{C}$. Sections were then rinsed and a tertiary incubation $\left(24 \mathrm{~h}\right.$ at $\left.4^{\circ} \mathrm{C}\right)$ with streptavidin 405 was performed to label the biotinylated secondary antibody. The final fluorescence detection channel assignment (excitation) was as follows: vGAT (405 nm), AnkG and NeuN (488 nm), PV (568 nm), and $\gamma 2(647 \mathrm{~nm})$. Note that AnkG and NeuN, which were both detected using mouse primary antibodies, were visualized in the same channel.

Microscopy. Image stacks $(512 \times 512$ pixels; $0.25 \mu \mathrm{m} z$-step $)$ were collected on an Olympus IX71 microscope controlled by SlideBook 5.0 (3I) and equipped with an Olympus spinning disk confocal, Hamamatsu C9100 EM-CCD, and LEP BioPrecision2 XYZ motorized stage using a $60 \times 1.42$ N.A. objective. Sampling was confined to layers $2-4$ (defined as $10-60 \%$ of the distance from pial surface to white matter) (Pierri et al., 1999) of PFC area 46 (Fig. 1) because the density of PV-IR cartridges is highest at 3 months and lowest in adult monkeys within these layers (Cruz et al., 2003); in contrast, the density of detectable PV-IR boutons was previously reported to be low in 3-month-old and highest in adult monkeys in these same layers (Erickson and Lewis, 2002). Sites were systematic randomly sampled using a grid of $190 \times 190 \mu \mathrm{m}^{2}$ with stacks collected from 20 randomly selected sites per section.

Image processing. Image stacks were taken using optimal exposures (greatest dynamic range/no saturated pixels), exposure corrected, deconvolved using the AutoQuant adaptive blind deconvolution algorithm, and segmented. Within each field, AISs were manually traced only if they were considered to be completely visualized as indicated by (1) continuity across $z$ planes; (2) a proximal end adjacent to a clearly defined NeuN-IR soma; (3) a distal end, defined by tapering of the AnkG signal to the width of an axon; and (4) the entire AIS was contained within a virtual sampling box. The virtual sampling box started and ended one $z$-plane from the top and bottom of the image stack, respectively, and had $x-y$ start/end coordinates that were located 20 pixels from any edge. AIS length was calculated by measuring the distance between unique points at the soma-hillock boundary, the centroid, and distal end of the AnkG-IR AIS profile.
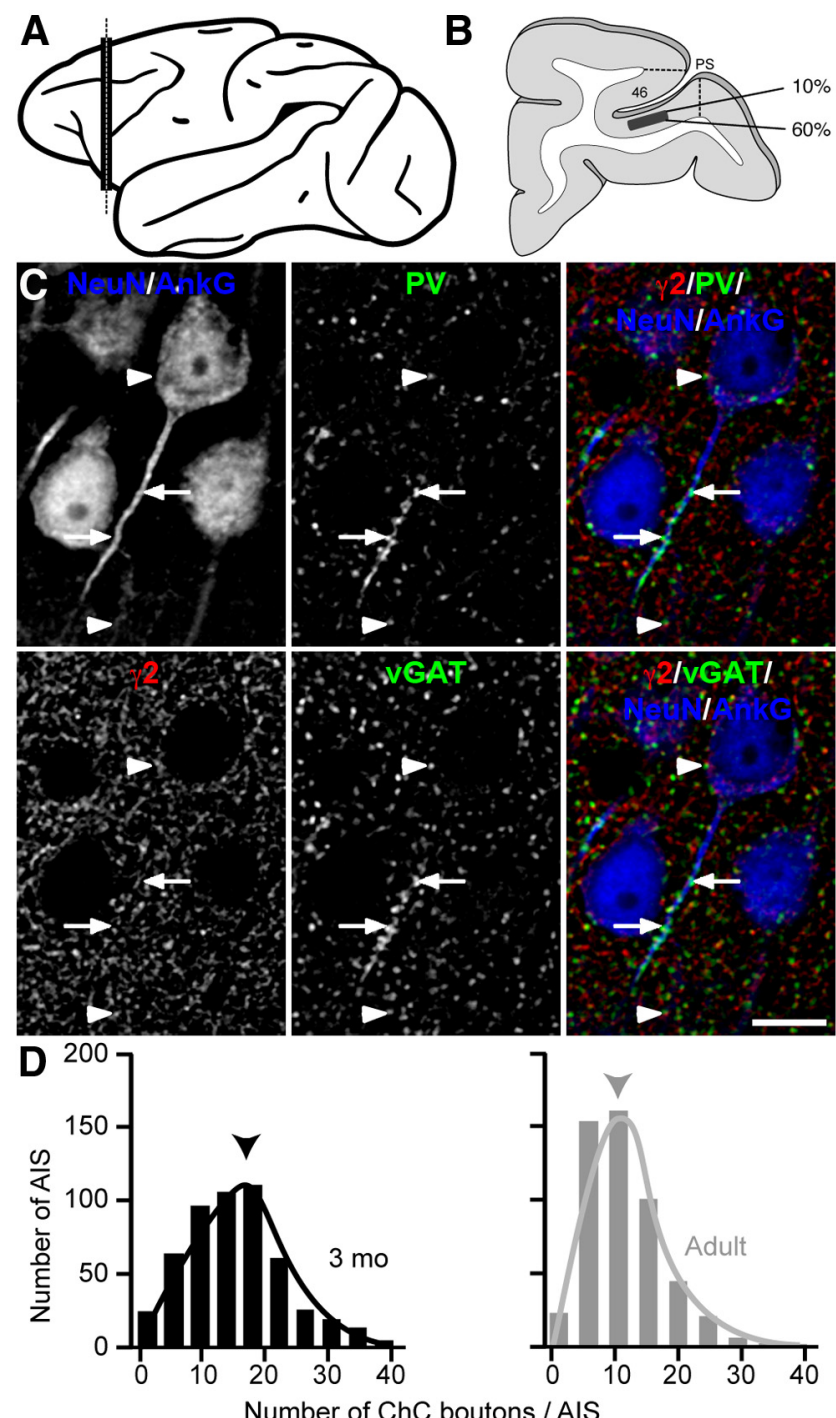

Figure 1. The mean number of PVChC boutons per AIS is decreased in adult compared with 3-month-old monkeys. $A$, Lateral view of monkey cortex showing the approximate location (black vertical bar) of the PFC where tissue sections for this study were taken. $\boldsymbol{B}$, Schematic of a typical coronal section containing the principal sulcus [(PS) area 46] at the position along the rostral-caudal axis designated by the dotted line in $\boldsymbol{A}$. The black rectangle designates the laminar location in the ventral bank of the PS where analyses were performed. C, Adult monkey section multilabeled for NeuN/AnkG, $\gamma 2$, PV, and vGAT. Single channel and merged projection images of a deconvolved image stack (five $z$-planes). Arrows, PVChC boutons; arrowheads, PVBC boutons. Scale bar, $10 \mu \mathrm{m}$. $\boldsymbol{D}$ Histogram of PVChC boutons per AIS in 3-month-old (black, mean 15.5) and adult (gray, mean 10.5) monkeys. Arrowheads point to respective means. A randomly selected 500 AIS from each age group is displayed.

Definitions of synaptic structures. Intensity/morphological segmentation was used to make object masks for each IR puncta, identified as small $\left(0.03-1 \mu \mathrm{m}^{3}\right)$, distinct fluorescing objects (Fish et al., 2011). Mask operations were then used to classify the different bouton populations. A GABAergic bouton was defined as a vGAT-IR puncta that was adjacent to a $\mathrm{GABA}_{\mathrm{A}}$ receptor $\gamma 2$-IR cluster. Because the width of a typical symmetric synaptic cleft is $\sim 20 \mathrm{~nm}$, the plasma membrane thickness is $\leq 10 \mathrm{~nm}$, and the lateral resolution of our system is $\sim 100 \mathrm{~nm}$ after postimage processing, only vGAT-IR puncta object masks that partially overlapped $\gamma 2$-IR cluster masks were considered adjacent and used for analysis. A PV bouton was defined as a PV object mask that contained the center of a vGAT object mask, which overlapped a $\gamma 2$ cluster object mask. Importantly, the presence of vGAT excludes the possibility that it is a thalamic 
bouton. PV boutons adjacent $\gamma 2$-IR clusters not associated with an AnkG-labeled AIS were classified as PVBC, whereas those adjacent $\gamma 2$-IR clusters within AnkG-labeled AISs were classified as PVChC. $\mathrm{ChC}_{\mathrm{PVneg}}$ boutons were defined as those GABAergic boutons adjacent $\gamma 2$-IR puncta within AnkG-labeled AISs that could not be represented by a PV object mask. Importantly, all GABAergic boutons that overlapped with $\gamma 2$-IR puncta within AnkG-labeled AISs were classified as being either a PVChC or a $\mathrm{ChC}_{\mathrm{PV} \text { neg }}$ bouton.

Statistics. Diagnostic statistics were used to confirm that the data were normally distributed. Independent $t$ tests were used to compare dependent measures between age groups. In all analyses, the statistics were performed on the mean values for individual monkeys $(N=3$ per age group) determined by first averaging data within stack, then averaging stack means within section, and finally averaging means across sections.

\section{Results \\ The density of GABAergic boutons does not differ between 3-month-old and adult monkeys}

The mean $( \pm S D)$ density of GABAergic boutons in layers $2-4$ of the PFC neuropil did not differ $\left(t_{(4)}=0.731, p=0.505\right)$ between 3 months $\left(0.045 \pm 0.006\right.$ boutons $\left./ \mu \mathrm{m}^{3}\right)$ and adult $(0.041 \pm$ 0.004 boutons $/ \mu \mathrm{m}^{3}$ ) animals. Because density measures may be confounded by age-related changes in PFC volume (Erickson and Lewis, 2002), we also determined the number of GABAergic boutons per NeuN-IR neuron, which did not differ $\left(t_{(4)}=0.939, p=\right.$ $0.401)$ between age groups.

\section{The mean number of PVChC boutons per AIS, but not of PVBC boutons per neuron, is lower in adult compared with 3-month-old monkeys}

The mean number of PVChC boutons per AIS was 32\% lower $\left(t_{(4)}=2.853, p=0.046\right)$ in adult $(10.5 \pm 1.7)$ relative to 3 -month-old (15.5 \pm 2.5$)$ monkeys (Fig. 1). Additionally, the magnitude of the difference between age groups was similar for ChC boutons that did not appear to contain PV $\left(\mathrm{ChC}_{\mathrm{PVneg}}\right)$ (Fig. 2). Given that the densities of GAT1 and PV-IR cartridges were previously reported to show different patterns of change both within the same layers and across layers during postnatal development (Cruz et al., 2003), we performed an additional analysis that took layer into account. Specifically, we separated sampling sites based on their laminar position into two groups: layers 2-superficial 3 and layers deep 3-4. Although the magnitude of the reduction in PVChC boutons was similar across layers, there was a twofold greater magnitude of reduction in $\mathrm{ChC}_{\mathrm{PVneg}}$ boutons in layers 2-superficial 3 compared with layers deep 3-4 (Fig. $2 B)$. In contrast to PVChC boutons, neither the number of PVBC boutons per NeuN-IR pyramidal neuron $\left(t_{(4)}=-0.201, p=\right.$ $0.851)$, nor the density of PVBC boutons in the neuropil $\left(t_{(4)}=\right.$ $-1.758, p=0.154)$, differed between 3-month-old (244 \pm 84 boutons/pyramidal neuron and $0.0101 \pm 0.0013$ boutons $/ \mu \mathrm{m}^{3}$, respectively) and adult ( $254 \pm 31$ boutons/pyramidal neuron and $0.0117 \pm 0.0008$ boutons $/ \mu \mathrm{m}^{3}$, respectively) monkeys.

To determine whether biased sampling could have contributed to the age-related decline in the mean number of $\mathrm{ChC}$ boutons per AIS, the robustness of our sampling was assessed. Although the coefficient of variation for the number of PVChC boutons per AIS within adult animals ranged from 75 to $129 \%$, the coefficient of error ranged from 5 to $10 \%$, indicating that the obtained means are robust estimates of the true population means. In addition, the difference in mean number of PVChC boutons per AIS between age groups was not accompanied by any change $\left(t_{(4)}=-1.289, p=0.267\right)$ in PV-IR cell body density, consistent with a prior report (Conde et al., 1996). These latter findings suggest that the lower number of PVChC boutons per AIS in the adult animals did not result from a postnatal loss of ChCs (Southwell et al., 2012).

A highly stringent criterion was used to define a PVChC bouton, raising the possibility that some PVChC to pyramidal AIS connections were excluded from the analysis. To ensure that the age-related reduction in mean number of PVChC boutons per AIS was not confounded by this possibility, the mean numbers of PV-IR, vGAT-IR, and $\gamma 2$-IR puncta that overlapped AIS were compared between age groups. The mean numbers of PV-IR and vGAT-IR puncta per AIS declined $\left(t_{(4)}=6.028, p=0.004\right.$ and $t_{(4)}=5.327, p=0.006$, respectively) between 3 -month-old $(19.2 \pm 2.0$ and $21.8 \pm 2.4$, respectively) and adult (11.8 \pm 0.6 and $14.2 \pm 0.7$, respectively) animals (Fig. 2). Likewise, the mean number of $\gamma 2$-IR puncta per AIS significantly declined $\left(t_{(4)}=3.577, p=0.023\right)$ between 3 -month-old $(26.7 \pm 4.8)$ and adult $(15.3 \pm 2.7)$ animals. The percentage declines in PV-IR, vGAT-IR, and $\gamma 2$-IR puncta per AIS, which ranged from 35 to $43 \%$, were similar to the $32 \%$ age-related decline in mean number of PVChC boutons per AIS.

AIS innervation by PVChCs was found to be highly variable in both 3-month-old (0-42 PVChC boutons per AIS) and adult (0-39 PVChC boutons per AIS) monkeys. Considering that the mean numbers of PVChC boutons per AIS in 3-month-old and adult animals were 15.5 and 10.5, respectively, these findings suggest that a large number of AIS might not be associated with a morphologically detectable cartridge and that the density of detectable PVChC cartridges might be significantly reduced between 3-month-old and adulthood. To assess cartridge density, PV-IR cartridges were identified qualitatively by their characteristic morphology and the number of PV-IR boutons per AIS was quantified. The PV channel was used to select PV-IR cartridges, and then the other channels (vGAT, $\gamma 2$, and AnkG/NeuN) were used to determine how many boutons were present in each cartridge. This approach revealed that PV-IR morphologically distinct cartridges were composed of 12 or more PVChC boutons. Of 500 randomly selected pyramidal cells from 3-month-old monkeys, the AIS of 320 (64\%) was innervated by $\geq 12$ boutons, whereas in adult monkeys the AIS of only 163/500 (32.6\%) pyramidal cells was innervated by $\geq 12$ boutons. This $49 \%$ decrease in the number of recognizable cartridges between 3 -month-old and adult monkeys is similar to the previously reported 55\% decline using a different method (Anderson et al., 1995; Cruz et al., 2003, 2009).

\section{The number of PVChC boutons per AIS is positively correlated with AIS size}

The mean length $\left(t_{(4)}=6.213, p=0.003\right)$ of AISs, as determined by AnkG immunoreactivity, was $\sim 33 \%$ shorter in adult $(19.7 \pm 1.4 \mu \mathrm{m})$ than in 3 -month-old $(29.1 \pm 2.3 \mu \mathrm{m})$ monkeys (Fig. $3 A$ ). In addition, the average AIS surface area determined by AnkG immunoreactivity was significantly less $\left(t_{(4)}=4.839, p=0.008\right)$ in adult $\left(132 \pm 8 \mu \mathrm{m}^{2}\right)$ than 3 -month-old $\left(202 \pm 24 \mu \mathrm{m}^{2}\right)$ monkeys (Fig. $3 B$ ). Furthermore, the number of PVChC boutons was positively correlated (3-month-old $r=0.764, p<0.001$; adult $r=0.735, p<0.001$ ) with AIS surface area within monkeys (Fig. $3 C$ ). Importantly, mean AnkG fluorescence intensity in AIS did not differ $\left(t_{(4)}=\right.$ $0.436, p=0.685)$ between adult $(2494 \pm 231)$ and 3 -monthold $(2554 \pm 67)$ monkeys, suggesting that group differences in 


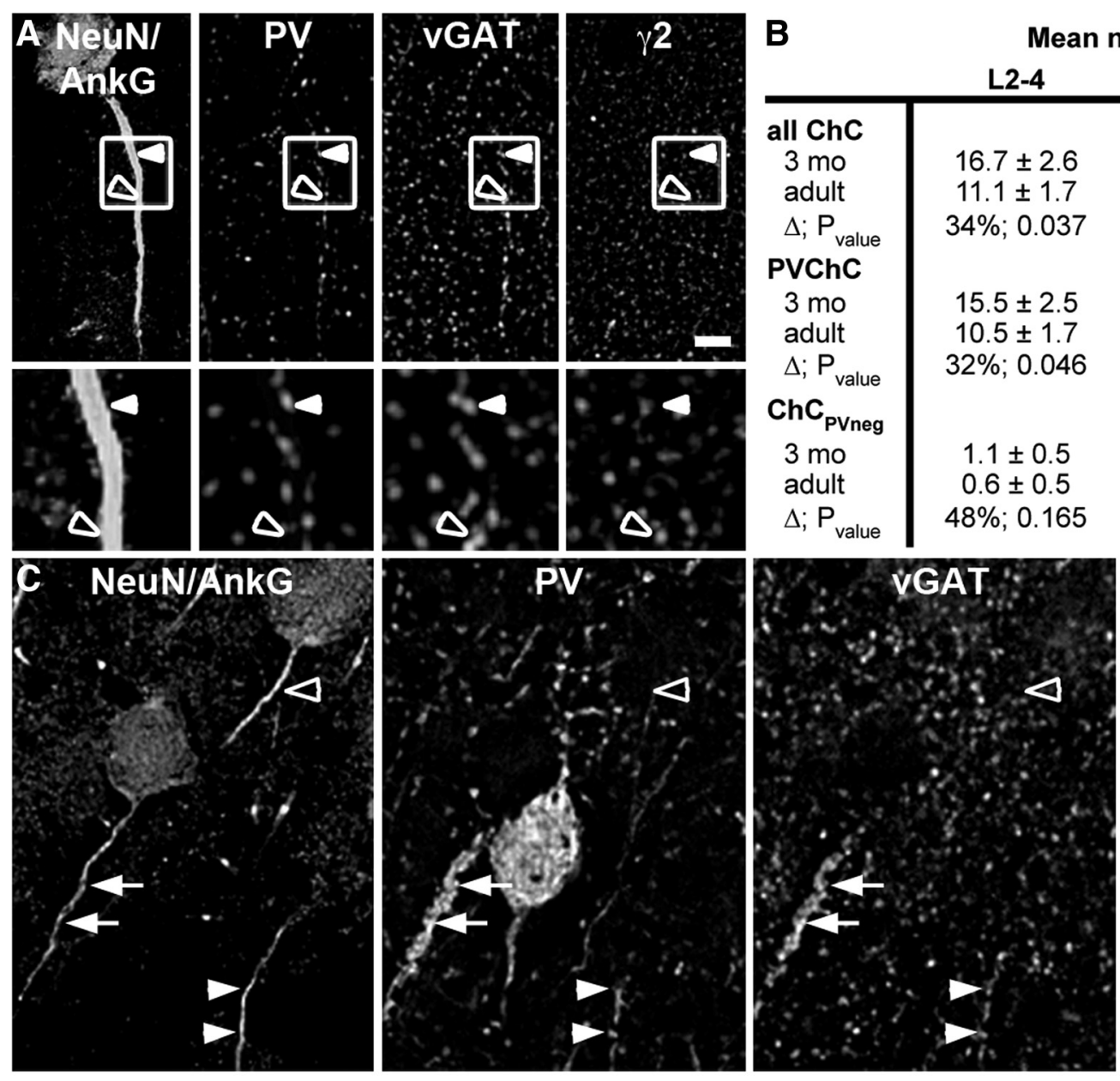

B Mean number of boutons

L2-3s L3d-4

\begin{tabular}{c|ccc}
\hline all ChC & & & \\
$3 \mathrm{mo}$ & $16.7 \pm 2.6$ & $17.9 \pm 2.1$ & $15.4 \pm 3.4$ \\
adult & $11.1 \pm 1.7$ & $11.4 \pm 2.7$ & $10.8 \pm 0.8$ \\
$\Delta ; \mathrm{P}_{\text {value }}$ & $34 \% ; 0.037$ & $36 \% ; 0.029$ & $30 \% ; 0.138$ \\
$\mathrm{PVChC}$ & & & \\
$3 \mathrm{mo}$ & $15.5 \pm 2.5$ & $16.5 \pm 2.2$ & $14.6 \pm 3.1$ \\
adult & $10.5 \pm 1.7$ & $10.8 \pm 2.6$ & $10.2 \pm 0.9$ \\
$\Delta ; \mathrm{P}_{\text {value }}$ & $32 \% ; 0.046$ & $34 \% ; 0.046$ & $30 \% ; 0.078$
\end{tabular}
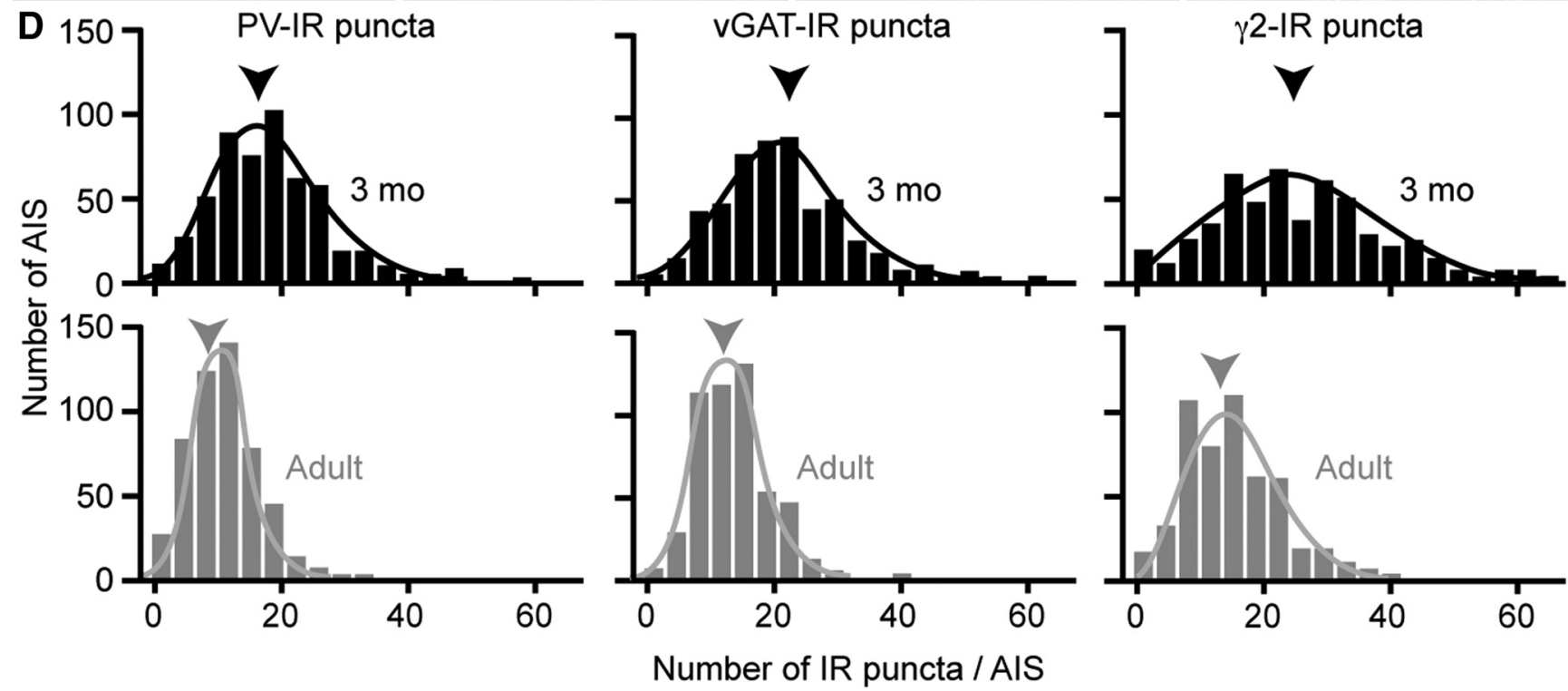

Figure 2. The mean number of puncta immunoreactive for PV, vGAT, or $\gamma 2$ overlapping AIS is decreased in adult compared with 3-month-old monkeys. A, Single channel images of a deconvolved image stack (five z-planes) from an adult monkey section multilabeled for NeuN/AnkG, PV, VGAT, and $\gamma 2$. Solid arrowhead, PVChC bouton; open arrowhead, ChC bouton with no visually detectable PV. A $3 \times$ zoom of the boxed region is shown below each channel. Scale bar, $10 \mu \mathrm{m}$. B, Table showing the mean number of (hC boutons per AlS (all ChC), ChC boutons classified as containing PV (PVChC), and $\mathrm{ChC}$ boutons classified as being PV-immunonegative ( $\mathrm{ChC}_{\mathrm{PVneg}}$ ). $\mathrm{C}$, Single channel images of a deconvolved image stack (five z-planes) of an adult monkey section multilabeled for NeuN/AnkG, PV, vGAT, and $\gamma 2$. Arrows, solid arrowheads, and the open arrowhead point to AIS differentially innervated by PVChCs. Scale bar, $10 \mu \mathrm{m}$. D, Histograms of PV (mean 3-month-old = 19.2, adult = 11.8), vGAT (mean 3-month-old = 21.8, adult = 14.2), and $\gamma 2$ (mean 3-month-old = 26.7, adult = 15.3) -IR puncta per AlS in 3-month-old (black) and adult monkeys (gray). Arrowheads point to respective means. A randomly selected 500 AIS from each age group is displayed. 


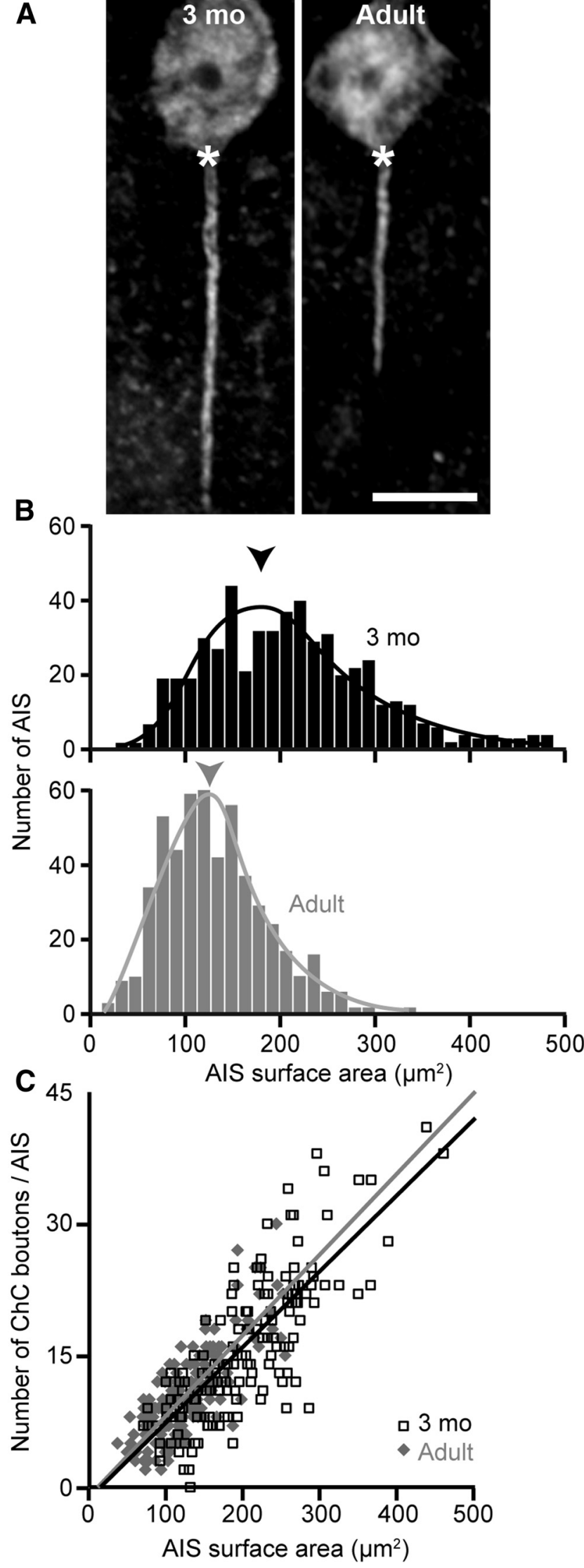

Figure 3. The number of PVChC boutons per AIS is positively correlated with AIS size. $\boldsymbol{A}$, NeuN and AnkG-labeled soma and AIS, respectively, from 3-month-old and adult monkeys. The selected neurons are representative of the length differences between age groups (see
Table 1. Relative protein levels in PVBC and PVChC boutons (PV and vGAT) adjacent to postsynaptic clusters $(\gamma 2)$ in 3-month-old and adult monkeys

\begin{tabular}{llllll}
\hline & \multicolumn{2}{l}{ PVBC } & & \multicolumn{2}{l}{ PVChC } \\
\cline { 2 - 3 } \cline { 5 - 6 } & 3 months & Adult & & 3 months & Adult \\
\hline PV & $3582 \pm 156$ & $6050 \pm 892$ & & $6261 \pm 1091$ & $6695 \pm 780$ \\
& $\left(t_{(2.12)}=-4.72, p=0.037\right)$ & & & $\left(t_{(4)}=-0.56, p=0.605\right)$ \\
VGAT & $5058 \pm 43$ & $6324 \pm 870$ & & $6176 \pm 80$ & $7102 \pm 1067$ \\
& $\left(t_{(4)}=-2.52, p=0.066\right)$ & & $\left(t_{(4)}=-1.50, p=0.208\right)$ \\
$\gamma 2$ & $1638 \pm 308$ & $2207 \pm 864$ & & $1702 \pm 357$ & $2042 \pm 894$ \\
& $\left(t_{(4)}=-1.08, p=0.343\right)$ & & $\left(t_{(4)}=-0.61, p=0.574\right)$ \\
\hline
\end{tabular}

AIS length and surface area were not confounded by changes in AnkG fluorescence.

Relative PV protein levels in PVBC boutons, but not in PVChC boutons, are significantly greater in adult compared with 3-month-old monkeys

The relative mean protein level of PV per PVBC bouton increased significantly by $69 \%$ between 3 -month-old and adulthood. This increase was accompanied by an increase in the relative mean protein level of vGAT (25\%) per PVBC bouton and in $\gamma 2(35 \%)$ relative protein levels in the corresponding postsynaptic clusters between 3-month-old and adulthood, although neither of these changes achieved statistical significance (Table 1). In contrast, relative mean protein levels of PV and vGAT per PVChC bouton, and $\gamma 2$ per postsynaptic cluster adjacent to a PVChC bouton were similar between the two age groups (Table 1).

\section{Discussion}

Prior reports of a decreased density of PVChC axon cartridges (Anderson et al., 1995; Cruz et al., 2003, 2009) and increased density of PV-IR boutons (Erickson and Lewis, 2002) over postnatal development in monkey PFC could represent inverse changes in bouton number and/or in PV protein levels per bouton. As reported here, the mean number of PVChC boutons per pyramidal neuron AIS was significantly 32\% lower in adult compared with 3-month-old monkeys, with no group differences in relative mean PV protein levels per PVChC bouton. In contrast, the density of PVBC boutons did not differ between age groups, but the relative mean PV protein levels in PVBC boutons were nearly twofold higher in adults than in 3-month-old monkeys. Thus, PVChC and PVBC appear to use fundamentally different mechanisms to achieve adult levels of innervation of their pyramidal cell targets.

The result that GABAergic bouton density in the PFC does not differ between 3-month-old and adult monkeys, along with previous reports that neither the density of symmetric synapses (Bourgeois et al., 1994) nor the density of GAT1-IR puncta (Erickson and Lewis, 2002) differed between these age groups suggest that GABAergic boutons are not pruned during postnatal development. In this context, our finding of an age-related reduction in PVChC boutons strongly suggests

Results: the number of PVChC boutons per AIS is positively correlated with AIS size). Asterisks designate the axon hillock. Scale bar, $10 \mu \mathrm{m}$. B, Histogram showing the surface area of 500 randomly selected AIS from each age group (3-month-old, black, mean 202; adult, gray, mean 132). Arrowheads point to respective means. C, Scatter plot of 150 randomly selected AIS from each age group ( 3 monkeys/group, $50 \mathrm{AIS} /$ monkey) showing the relationship between AIS surface area and the number of PVChC boutons. 
that the pruning of these boutons is cell-type-specific and not detectable when all GABAergic boutons are assessed without regard to cell type.

In this study, AIS mean length and average surface area were $\sim 33 \%$ less in adult than in 3-month-old monkeys, consistent with previous reports (Cruz et al., 2009). Recent studies have shown that AIS length/location depend, at least in part, on network activity (Grubb et al., 2011). Interestingly, we found that the number of PVChC boutons was positively correlated with AIS surface area. In concert, these findings suggest that the number of $\mathrm{ChC}$ inputs onto a given pyramidal neuron is also regulated by network activity.

The distinctiveness of a developmental reduction in the number of PVChC boutons may be related to their unique role in innervating the AIS of pyramidal neurons where they are positioned to regulate action potentials. Interestingly, we found that the number of $\mathrm{ChC}$ boutons associated with an AIS is highly variable across AIS in both 3-month-old (0-42 PVChC boutons per AIS) and adult (0-39 PVChC boutons per AIS) (Fig. 1D) monkeys, similar to prior electron microscopy studies in adult primates (DeFelipe et al., 1985; Inda et al., 2007). These findings raise the possibility that the degree of action potential regulation by ChCs differs across subtypes of pyramidal neurons. Consistent with this idea, the mean number of inputs to the AIS is greatest in callosal-projecting pyramidal cells, intermediate in pyramidal cells providing ipsilateral cortical projections, and lowest in corticothalamic pyramidal neurons in monkey neocortex (Farinas and DeFelipe, 1991). However, even among pyramidal neurons that share the same projection target, the number of $\mathrm{ChC}$ inputs to the AIS shows considerable variability (Farinas and DeFelipe, 1991). These findings, and those of this study, reveal a marked heterogeneity in the need for $\mathrm{ChC}$ regulation of pyramidal neurons, across and within subclasses of pyramidal cells, and apparently across development for the same pyramidal cells. Given the reported roles of $\mathrm{ChC}$ inputs of either hyperpolarizing or depolarizing pyramidal cells based on levels of network activity (Woodruff et al., 2011) or of blocking retrograde action currents (Dugladze et al., 2012), why some pyramidal cells need a high level of these types of regulation and others do not is an important question for future studies.

In contrast to PVChC, relative PV protein levels in PVBC boutons were approximately twofold higher in adult compared with 3-month-old monkeys. In concert with our finding that PVBC bouton density did not differ between age groups, these results suggest that the previously reported increase in PV-IR puncta density across postnatal development in monkey PFC results from an age-related rise in PV protein levels per PVBC bouton and thus an increase in their detectability by standard light microscopy. Although the physiological role of $\mathrm{Ca}^{2+}$ buffering by PV is still unclear, an increase in bouton PV levels decreases the duration of $\mathrm{Ca}^{2+}$ transients that influence GABA release (Collin et al., 2005). Thus, an increase in PVBC bouton PV levels could result in greater bouton-specific regulation over GABA release with age.

The distinct differences in the maturation of PVBC and PVChC boutons may provide insight into the nature or timing of the reported alterations in these types of boutons in schizophrenia. For example, the density of ChC cartridges detectable by GAT1 immunoreactivity is $40 \%$ lower in the PFC of schizophrenia subjects relative to matched comparison subjects (Woo et al., 1998; Pierri et al., 1999). This difference could represent an excessive developmental elimination of $\mathrm{ChC}$ boutons. In addition, lower levels of PV protein in putative PVBC boutons in schizophrenia (J. R. Glausier, K. N. Fish, and D. A. Lewis, unpublished observation) might represent a disease-related impairment in the normal developmental rise of PV levels in these boutons observed in this study. Together, these alterations in perisomatic inhibitory regulation of pyramidal cell function might contribute to the emergence of PFCmediated cognitive disturbances well before the clinical onset of schizophrenia during late adolescence or early adulthood (Reichenberg et al., 2010).

\section{References}

Anderson SA, Classey JD, Condé F, Lund JS, Lewis DA (1995) Synchronous development of pyramidal neuron dendritic spines and parvalbuminimmunoreactive chandelier neuron axon terminals in layer III of monkey prefrontal cortex. Neuroscience 67:7-22. CrossRef Medline

Bourgeois JP, Goldman-Rakic PS, Rakic P (1994) Synaptogenesis in the prefrontal cortex of rhesus monkeys. Cereb Cortex 4:78-96. CrossRef Medline

Chaudhry FA, Reimer RJ, Bellocchio EE, Danbolt NC, Osen KK, Edwards RH, Storm-Mathisen J (1998) The vesicular GABA transporter, VGAT, localizes to synaptic vesicles in sets of glycinergic as well as GABAergic neurons. J Neurosci 18:9733-9750. Medline

Collin T, Chat M, Lucas MG, Moreno H, Racay P, Schwaller B, Marty A, Llano I (2005) Developmental changes in parvalbumin regulate presynaptic $\mathrm{Ca}^{2+}$ signaling. J Neurosci 25:96-107. CrossRef Medline

Condé F, Lund JS, Lewis DA (1996) The hierarchical development of monkey visual cortical regions as revealed by the maturation of parvalbuminimmunoreactive neurons. Brain Res Dev Brain Res 96:261-276. CrossRef Medline

Cruz DA, Eggan SM, Lewis DA (2003) Postnatal development of presynaptic and postsynaptic GABA markers at chandelier cell connections with pyramidal neurons in monkey prefrontal cortex. J Comp Neurol 465:385-400. CrossRef Medline

Cruz DA, Lovallo EM, Stockton S, Rasband M, Lewis DA (2009) Postnatal development of synaptic structure proteins in pyramidal neuron axon initial segments in monkey prefrontal cortex. J Comp Neurol 514:353-367. CrossRef Medline

DeFelipe J, Hendry SH, Jones EG, Schmechel D (1985) Variability in the terminations of GABAergic chandelier cell axons on initial segments of pyramidal cell axons in the monkey sensory-motor cortex. J Comp Neurol 231:364-384. CrossRef Medline

DeFelipe J, Hendry SH, Jones EG (1989) Visualization of chandelier cell axons by parvalbumin immunoreactivity in monkey cerebral cortex. Proc Natl Acad Sci U S A 86:2093-2097. CrossRef Medline

Dugladze T, Schmitz D, Whittington MA, Vida I, Gloveli T (2012) Segregation of axonal and somatic activity during fast network oscillations. Science 336:1458-1461. CrossRef Medline

Erickson SL, Lewis DA (2002) Postnatal development of parvalbumin- and GABA transporter-immunoreactive axon terminals in monkey prefrontal cortex. J Comp Neurol 448:186-202. CrossRef Medline

Fariñas I, DeFelipe J (1991) Patterns of synaptic input on corticocortical and corticothalamic cells in the cat visual cortex: II. The axon initial segment. J Comp Neurol 304:70-77. CrossRef Medline

Fish KN, Sweet RA, Lewis DA (2011) Differential distribution of proteins regulating GABA synthesis and reuptake in axon boutons of subpopulations of cortical interneurons. Cereb Cortex 21:2450-2460. CrossRef Medline

Fritschy JM, Mohler H (1995) GABA A-receptor heterogeneity in the adult rat brain: differential regional and cellular distribution of seven major subunits. J Comp Neurol 359:154-194. Medline

Grubb MS, Shu Y, Kuba H, Rasband MN, Wimmer VC, Bender KJ (2011) Short- and long-term plasticity at the axon initial segment. J Neurosci 31:16049-16055. CrossRef Medline

Guo C, Stella SL Jr, Hirano AA, Brecha NC (2009) Plasmalemmal and vesicular gamma-aminobutyric acid transporter expression in the developing mouse retina. J Comp Neurol 512:6-26. CrossRef Medline

Hoftman GD, Lewis DA (2011) Postnatal developmental trajectories of neural circuits in the primate prefrontal cortex: identifying sensitive periods for vulnerability to schizophrenia. Schizophr Bull 37:493503. CrossRef Medline 
Inda MC, Defelipe J, Muñoz A (2007) The distribution of chandelier cell axon terminals that express the GABA plasma membrane transporter GAT-1 in the human neocortex. Cereb Cortex 17:2060-2071. CrossRef Medline

Kägi U, Berchtold MW, Heizmann CW (1987) $\mathrm{Ca}^{2+}$-binding parvalbumin in rat testis: characterization, localization, and expression during development. J Biol Chem 262:7314-7320. Medline

Lewis DA, Lund JS (1990) Heterogeneity of chandelier neurons in monkey neocortex: corticotropin-releasing factor- and parvalbuminimmunoreactive populations. J Comp Neurol 293:599-615. CrossRef Medline

Mullen RJ, Buck CR, Smith AM (1992) NeuN, a neuronal specific nuclear protein in vertebrates. Development 116:201-211. Medline

Pierri JN, Chaudry AS, Woo TU, Lewis DA (1999) Alterations in chandelier neuron axon terminals in the prefrontal cortex of schizophrenic subjects. Am J Psychiatry 156:1709-1719. Medline

Reichenberg A, Caspi A, Harrington H, Houts R, Keefe RS, Murray RM, Poulton R, Moffitt TE (2010) Static and dynamic cognitive deficits in childhood preceding adult schizophrenia: a 30-year study. Am J Psychiatry 167:160-169. CrossRef Medline

Southwell DG, Paredes MF, Galvao RP, Jones DL, Froemke RC, Sebe JY, Alfaro-Cervello C, Tang Y, Garcia-Verdugo JM, Rubenstein JL, Baraban SC, Alvarez-Buylla A (2012) Intrinsically determined cell death of developing cortical interneurons. Nature 491:109-113. CrossRef Medline

Szentágothai J, Arbib MA (1974) Conceptual models of neural organization. Neurosci Res Program Bull 12:305-510. Medline

Wang X, Sun QQ (2012) Characterization of axo-axonic synapses in the piriform cortex of Mus musculus. J Comp Neurol 520:832-847. CrossRef Medline

Woo TU, Whitehead RE, Melchitzky DS, Lewis DA (1998) A subclass of prefrontal gamma-aminobutyric acid axon terminals are selectively altered in schizophrenia. Proc Natl Acad Sci USA 95:53415346. CrossRef Medline

Woodruff AR, McGarry LM, Vogels TP, Inan M, Anderson SA, Yuste R (2011) State-dependent function of neocortical chandelier cells. J Neurosci 31:17872-17886. CrossRef Medline 